\title{
REFERENCES
}

1. P. Erdös, On the asymptotic density of the sum of two sequences, Ann. of Math. vol. 43 (1942) pp. 65-68.

2. H. B. Mann, $A$ proof of the fundamental theorem on the density of sums of sets of positive integers, Ann. of Math. vol. 43 (1942) pp. 523-527.

3. Emil Artin and Peter Scherk, On the sum of two sets of integers, Ann. of Math. vol. 44 (1943) pp. 138-142.

New YoRK UNIVERSITY

\section{CERTAIN BEST POSSIBLE RESULTS IN THE THEORY OF SCHNIRELMANN DENSITY}

\section{BENJAMIN LEPSON ${ }^{1}$}

Let $A$ be a set of distinct non-negative integers, and $A(n)$ the number of positive integers not greater than $n$ in $A$. Then the Schnirelmann density $\alpha$ of $A$, referred to below simply as density, is defined as

$$
\alpha=\text { g.l.b. } \frac{A(n)}{n} \text {. }
$$

If $A$ and $B$ are two such sets, the set $C=A+B$ consists of all distinct sums $a+b$ with $a$ in $A$ and $b$ in $B$. The densities of $A, B$, and $C$ will be denoted by $\alpha, \beta$, and $\gamma$ respectively.

The $\alpha+\beta$ hypothesis, first proved by Mann [3], ${ }^{2}$ states that, if $A$ and $B$ each contain 0 , then

$$
\gamma \geqq \min (1, \alpha+\beta) \text {. }
$$

Erdös and Niven [2] state that (1) is "a best possible result" without giving details. It is shown below that, under the above conditions, and for any pair $(\alpha, \beta)$, there exist sets for which the equality sign holds in (1).

THEOREM 1. Let $\alpha$ and $\beta$ be any real numbers such that

$$
0 \leqq \alpha \leqq 1 \text { and } 0 \leqq \beta \leqq 1 .
$$

Then there exist sets $A$ and $B$, each containing 0 and of densities $\alpha$ and

Presented to the Society, October 29, 1949; received by the editors August 12, 1949.

1 Atomic Energy Commission pre-doctoral fellow.

2 Numbers in brackets refer to the references cited at the end of the paper. 
$\beta$ respectively, such that the density $\gamma$ of $C$ is given by

$$
\gamma=\min (1, \alpha+\beta) \text {. }
$$

Proof. For every $n>0$, let $a_{n}$ be the least positive integer not greater than $\alpha \cdot n !$. Then

$$
0 \leqq a_{n+1}-a_{n} \leqq(n+1) !-n ! .
$$

Define $A$ as the set consisting of 0,1 , and each of the (possibly vanishing) blocks $n !+1, n !+2, \cdots, n !+a_{n+1}-a_{n}$. By (3), no two blocks have an element in common; therefore

$$
\frac{A(n !)}{n !}=\frac{1+\sum_{j=1}^{n-1}\left(a_{j+1}-a_{j}\right)}{n !}=\frac{a_{n}}{n !} \geqq \alpha .
$$

Since

$$
\lim _{n \rightarrow \infty} \frac{a_{n}}{n !}=\alpha
$$

and

$$
\frac{A(n)}{n} \geqq \frac{A(m !)}{m !} \geqq \alpha
$$

for some $m$ depending upon $n$, we see that the density of $A$ is $\alpha$. Define $b_{n}$ and $B$ similarly in terms of $\beta$.

Let $C=A+B$ have density $\gamma$, and let $c \leqq n$ ! belong to $C$. Then $c=a+b$ where $a \leqq n !$ and $b \leqq n !$, so that, from the construction of $A$ and $B, a \leqq(n-1) !+a_{n}-a_{n-1}$ and $b \leqq(n-1) !+b_{n}-b_{n-1}$. Therefore

$$
\begin{aligned}
c & \leqq 2(n-1) !+a_{n}+b_{n}-a_{n-1}-b_{n-1} \\
& \leqq 2(n-1) !+a_{n}+b_{n}
\end{aligned}
$$

which implies

$$
C(n !) \leqq 2(n-1) !+a_{n}+b_{n}
$$

from which it follows that

$$
\lim _{n \rightarrow \infty} \frac{C(n !)}{n !} \leqq \alpha+\beta
$$

and finally

$$
\gamma \leqq \alpha+\beta .
$$


Combining (1) and (6), we have (2). ${ }^{3}$

One may ask whether it is possible to weaken the condition that $A$ and $B$ both contain 0 and still to have (1). In this connection, we have the following result.

THEOREM 2. Let $E$ and $F$ be finite sets of distinct non-negative integers. If, whenever $A$ contains $E$ and $B$ contains $F,(1)$ holds, then $E$ and $F$ both contain 0.

Proof. Suppose that $E$ does not contain 0 . Let $N$ be the largest integer in $E$ or $F$. Define $A$ as the set of positive integers congruent to any of the integers $1,2, \cdots, N(\bmod 2 N+1)$ and $B$ as the set of non-negative integers congruent to any of the integers $0,1,2, \cdots$, $N(\bmod 2 N+1)$. Then

$$
\alpha=\frac{N}{2 N+1}, \quad \beta=\frac{1}{2}, \quad \gamma=\frac{2 N}{2 N+1}
$$

so that

$$
\gamma<\alpha+\beta<1
$$

which contradicts (1). Similarly, $F$ contains 0 .

Finally, we note that (1) cannot be improved by requiring $A$ and $B$ to contain certain finite sets in addition to 0 . This follows immediately from Theorem 1, with the observations that the densities of the sets $A$ and $B$ used in the proof would be unchanged by the addition of finite sets, and that (4) would remain valid for sufficiently large $n$, implying (5) and (6).

\section{REFERENCES}

1. E. Artin and P. Sherk, On the sum of two sets of integers, Ann. of Math. vol. 44 (1943) pp. 138-142.

2. P. Erdös and I. Niven, The $\alpha+\beta$ hypothesis and related problems, Amer. Math. Monthly vol. 53 (1946) pp. 314-317.

3. H. B. Mann, $A$ proof of the fundamental theorem on the density of sums of sets of positive integers, Ann. of Math. vol. 43 (1942) pp. 523-527.

\section{Columbia University}

I It is possible, by a more detailed argument, to prove (2) directly without the use of (1). 